\title{
Orbit of Galactic Globular Cluster of NGC 7089, NGC 7099, NGC 1851, Pal 1, and Pal 2 Around the Milky Way Galaxy Constructed from the Gaia EDR3
}

Raj K. Pradhan, Sabina Gautam, Madhu S. Paudel, and Prajwal R. Kafle

Journal of Nepal Physical Society

Volume 7, No 3, 2021

(Special Issue: ANPA Conference, 2021)

ISSN: 2392-473X (Print), 2738-9537 (Online)

\section{Editors:}

Dr. Nabin Malakar (Editor in chief)

Worcester State University

Dr. Pashupati Dhakal

Thomas Jefferson National Accelerator Facility, USA

Dr. Arjun Dahal

University of South Alabama, USA

Dr. Chiranjivi Lamsal

SUNY Platsburgh, USA

Dr. Dilli Raj Paudyal

University of Regina, Canada

Managing Editor:

Dr. Binod Adhikari

St. Xavier's College, Kathmandu, Nepal

JNPS, 7 (3), $72-76$ (2021)

DOI: http://doi.org/10.3126/jnphyssoc.v7i3.42515

Published by: Nepal Physical Society

P.O. Box: 2934

Tri-Chandra Campus

Kathmandu, Nepal

Email: npseditor@gmail.com

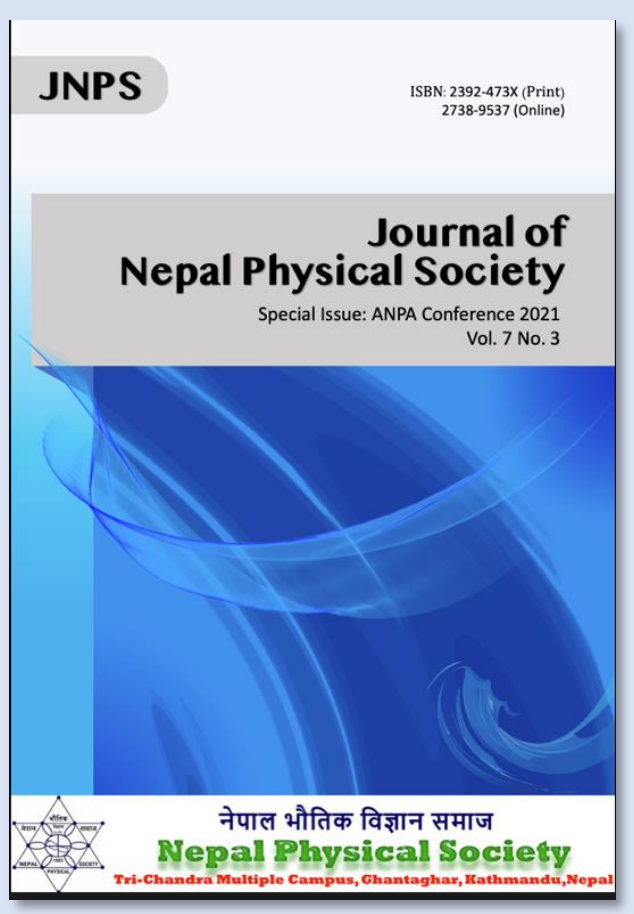




\title{
Orbit of Galactic Globular Cluster of NGC 7089, NGC 7099, NGC 1851, Pal 1, and Pal 2 Around the Milky Way Galaxy Constructed from the Gaia EDR3
}

\author{
Raj K Pradhan,, , 2, a) Sabina Gautam, ${ }^{3}$ Madhu S Paudel, ${ }^{3}$ and Prajwal R Kafle ${ }^{4}$ \\ ${ }^{1)}$ Central Department of Physics, Tribhuvan University, Kirtipur-44618, Nepal \\ 2) Pokhara Astronomical Society, Pokhara, Nepal \\ ${ }^{3)}$ Tri-Chandra Multiple Campus, Ghantaghar-44600, Kathmandu, Nepal \\ 4) International Centre for Radio Astronomy Research (ICRAR), The University of Western Australia, 35 Stirling Highway, \\ Crawley, WA 6009, Australia \\ a) Corresponding author: rj.physics55@gmail.com
}

\begin{abstract}
We report that we estimated the mean proper motion, the full space velocity of five Globular clusters, Pal 1, Pal2, NGC 1815, NGC 7089, and NGC 7099, using Gaia Early Data Release 3 (Gaia EDR3). We focus on these five clusters to measure proper motion, internal dispersion, and dynamical properties. Also, we have integrated the orbits using the potential of MWPotential2014 for five backyards Gyr. We have derived energy, angular momentum, and orbital properties for each cluster. In addition, we find that there are four metal-poor GCs with metallicity less than -1.1 dex and only one metal-rich GCs with metallicity greater than -1.0 dex. We estimate the distance modulus of these five globular clusters and examine isochrone fitting to the color-magnitude diagram from the PAdova and TRieste Stellar Evolution Code (PARSEC). Among five globular clusters, we have found that the NGC 7099 is the oldest globular cluster age of $\sim 13 \mathrm{Gyr}$ with metallicity ([Fe/H]) of 2.19, whereas the youngest globular cluster we find in our sample is Pal 1 with metallicity $([\mathrm{Fe} / \mathrm{H}])$ of -0.65 and age of $\sim 8 \mathrm{Gyr}$.
\end{abstract}

Keywords: Kinematics and Dynamics; globular clusters; general - Galaxy;

Received: 31 August 2021; Revised: 30 December 2021; Accepted: 1 January 2022

\section{INTRODUCTION}

Globular clusters (GCs) have a long history in the Galaxy which helps us to uncover the mystery of evolutionary history. They are gravitationally bounded, containing a compact form of stars in general. They consist of the oldest cluster's members are comparable to the age of the Universe. It is thought that GCs in the inner region of halo in the Milky Way formed in situ whereas the outer GCs formed through accretion along with dwarf galaxies. In the Milky Way, so far, it has been 170 globular clusters [1] discovered with derived precise proper motion and have more than 100 clusters with dispersion profile estimation and 162 globular clusters with a more accurate distance that estimated by [2]. Although, we do not know about their origin and formation history in detail which is still an open question in astrophysics. This paper is organized as follows. First, in Data and Method Section, we will discuss data reduction, filtering, and the method used. Next, the results of the finding with discussion are presented in the Results and Discussion Section. A summary of our main findings will be presented in the last section.

\section{DATA AND METHOD}

We have used the Gaia early data released 3 (Gaia EDR3) [3] which provides more accurate and precise proper motions and parallaxes than the previous data set of Gaia survey. It consists of more than 1.8 billion sources with a photometric band of $\mathrm{G}$ down to 21 magnitude. In addition, Gaia EDR3 possesses the previous line-of-sight velocity, with more than seven million stars. Furthermore, in the Gaia EDR3 catalogue, the parallax precision is increased by $30 \%$, whereas the proper motion precision is 
improved by a factor of 2 . Thanks to the Gaia survey for providing unprecedented data set for mapping the detailed picture of the three-dimensional kinematics of our home galaxy.

Our main aim is to study several kinematic properties and isochrone fitting to color-magnitude diagram (CMD) of the selected five globular clusters, NGC 1851, NGC 7089, and NGC 7099, Pal 1, and Pal2; Among these clusters, NGC 1851, NGC 7089, NGC 7099 have a large number of stars, whereas Pal 1 and Pal 2 have less number of stars.

In order to to filter the data set, we have used the following astrometric parameters recommended by [4]. Using this filtered data catalouge, we have computed proper motion (PM) and internal PM dispersion using publicly available code at github; https://github.com/GalacticDyn amics-Oxford/GaiaTools. There are the following steps we performed;

- We have taken five different clusters from the Gaia archive. The selected GCs have retained within one degree around the galactic center.

- To retain a clean subset of cluster's members, we exclude star having the following astrometric parameters (a) $\mathrm{G}<13$, (b) RUWE $>1.15$, (c) astrometric_excess_noise_sig $>2$,

(d) ipd_gof_harmonic_amplitude $>\exp [0.18$ (G - 33)], (e) ipd_frac_multi_peak > 2, (f) visibility_period_used $<10$, and

(g) phot_bp_rp_excess_factor $<1.3+0.06$ (bp_rp $)^{2}$

- To remove nearby stars along line-of-sight, we use $\bar{\omega}<1 / D+3 \varepsilon_{\bar{\omega}}$, where $D$ is the distance of the cluster from the Sun and $\varepsilon_{\bar{\omega}}$ uncertainty in parallax.

After applying the above selection criteria, we obtain a clean data set for further estimating our targeted globular clusters' mean proper motion and orbital velocities. There is various approach to identify cluster members. One of the powerful approach to identify them is the Gaussian Mixture model [5] [2] [1] and Bayesian approach [6]. Using this approach, we find highly probable stars associated with globular clusters. Nevertheless, we focused on dense clusters finding internal proper motion dispersion profile after using recommended filters [7] [1].

In this paper, we use two-dimensional Gaussian Kernels, which are applicable for three clusters (NGC 1851, NGC 7089, and NGC 7099) for determining a peak value of positions; although we calculate the mean proper motion of all clusters using Mixture model.

Due to the lack of radial velocity parameter in our sample, we have adopted this parameter from [5] and more accurate distances from [2]. We further estimated the space velocity of our selected five Globular clusters (Pal 1, Pal 2, NGC 1851, NGC 7089, and NGC 7099). To do this, first, we transfer the heliocentric coordinate system into Galactocentric in the Cartesian coordinate system using Astropy. The solar motions with local circular velocity are taken as a default of Astropy where the values of $U_{\odot}, V_{\odot}+V_{0}, W_{\odot}$ are $(12.9,245.6,7.78) \mathrm{kms}^{-1}$ [8] where $U, V, W$ represent the velocities along the direction of Galactic center, the tangential direction of disk rotation, and direction towards North Galactic plane, and $V_{0}$ be the local circular velocity. Assuming MWPotential2014 potential [9] we have estimated energy, angular momentum, eccentricity, and apocentric and pericentric distance from the Galactic center. We have taken the distance [2], radial velocity [5], metallicity [10] and position [1] for the study of orbital properties of five globular clusters.

\section{RESULTS AND DISCUSSIONS}

Table 1 presents the derived PM and other required parameters to study the kinematics of all five globular clusters. The globular cluster, Pal 2, is located at a distance of $26.174 \pm 1.285 \mathrm{kpc}$, the farthest clusters sample, with a radial velocity of $-135.97 \pm 1.55 \mathrm{kms}^{-1}$. In addition, this cluster has a low eccentric orbit, i.e., $e=0.304 \pm 0.009$ compared to other four clusters.

In Fig. 1, we plot different five globular clusters' positions, proper motion, color-magnitude diagram (CMD). This figure shows that three clusters (NGC 1851, NGC7089, and NGC 7099) are highly dense, whereas two clusters (Pal 1 and $\mathrm{Pal}$ 2) are less dense stars. Each figure of the bottom right plane shows the isochrone fitting of all five globular clusters. Fig. 2 shows the sample orbit of NGC 7099. The integrated orbits are 5 Gyr backward, assuming the same potential, i.e., MWPotential2014. Table 2 presents the obtained value of fundamental parameters of the GC sample using PARSEC isochrone with reddening and distance modulus. For isochrone fitting analysis, we used different literature to acquire a good fitting for globular clusters. In this study, we obtained the CMD of NGC 7099 has a good fit with metallicity $([\mathrm{Fe} / \mathrm{H}])$ of 2.19 with $E(B-V)=0.033$ and distance modulus of 14.80 . This value is in good agreement with other literature. Likewise, we have acquired the color-magnitude diagram (CMD) of NGC 7089 and NGC 1851, which is a good fit with metallicity of -1.65 and -1.18 with $E(B-V)=$ 0.05 and 0.02 and distance modulus of 15.35 and 15.5, respectively. In the case of Pal 1 and Pal 2, it was a challenging task to fit the isochrone because we did not find robust data set of Main-Sequence stars. Furthermore, we obtained robust data set of the GC member using the Gaussian Mixture model (GMM) based on [1]. Finally, we derived each parameter's uncertainty using the error prorogation method. For this, we used the Monte Carlo simulation method for 100 integration. Also, we run em- 
TABLE I. This table presents the estimated parameters of all five globular clusters.

\begin{tabular}{|c|c|c|c|c|c|c|c|c|c|c|c|c|c|}
\hline Cluster & $\begin{array}{c}\text { RA } \\
\text { DEC } \\
\text { (deg) }\end{array}$ & $\begin{array}{c}d \\
\sigma_{d} \\
(\mathrm{kpc})\end{array}$ & $\begin{array}{c}R_{v} \\
\sigma_{R_{v}} \\
\left(\mathrm{kms}^{-} 1\right)\end{array}$ & $\begin{array}{c}\mu_{\alpha *} \\
\sigma_{\mu_{\alpha^{*}}} \\
\left(\mathrm{mas} \mathrm{yr}^{-1}\right)\end{array}$ & $\begin{array}{c}\mu_{\delta} \\
\sigma_{\mu_{\delta}} \\
\left(\mathrm{mas} \mathrm{yr}^{-1}\right)\end{array}$ & $\rho_{\mu_{\alpha}}$ & $\begin{array}{c}x \\
\sigma_{x} \\
(\mathrm{kpc})\end{array}$ & $\begin{array}{c}y \\
\sigma_{y} \\
(\mathrm{kpc})\end{array}$ & $\begin{array}{c}z \\
\sigma_{z} \\
(\mathrm{kpc})\end{array}$ & $\begin{array}{c}v_{x} \\
\sigma_{x} \\
\left(\mathrm{kms}^{-1}\right)\end{array}$ & $\begin{array}{c}v_{y} \\
\sigma_{y} \\
\left(\mathrm{kms}^{-1}\right)\end{array}$ & $\begin{array}{c}v_{z} \\
\sigma_{z} \\
\left(\mathrm{kms}^{-1}\right)\end{array}$ & $\begin{array}{l}\text { ecc } \\
\sigma_{e c c}\end{array}$ \\
\hline \multirow[t]{2}{*}{ Pal 1} & 53.333 & 11.176 & -75.72 & 0.183 & 0.282 & 0.000 & -14.928 & 8.103 & 3.69 & 65.158 & 199.729 & -25.083 & 0.481 \\
\hline & 79.581 & 0.324 & 0.29 & 0.470 & 0.470 & & 0.179 & 0.213 & 0.096 & 1.475 & 1.326 & 1.685 & 0.005 \\
\hline \multirow[t]{2}{*}{ Pal 2} & 71.525 & 26.174 & -135.97 & 0.799 & -1.965 & 0.003 & -33.565 & 4.242 & -4.031 & 110.951 & -2.872 & 7.462 & 0.304 \\
\hline & 31.381 & 1.285 & 1.55 & 0.152 & 0.151 & & 1.273 & 0.212 & 0.203 & 2.517 & 12.026 & 4.475 & 0.009 \\
\hline \multirow[t]{2}{*}{ NGC 1851} & 78.528 & 11.951 & 321.4 & 2.243 & -0.545 & -0.001 & -12.345 & -8.823 & -6.822 & -80.785 & -74.55 & -82.686 & 0.702 \\
\hline & -40.047 & 0.134 & 1.55 & 0.114 & 0.114 & & 0.047 & 0.098 & 0.076 & 1.431 & 1.669 & 1.561 & 0.004 \\
\hline \multirow[t]{2}{*}{ NGC 7089} & 323.363 & 11.693 & -3.78 & 2.808 & -2.499 & 0.011 & -2.476 & 7.617 & -6.832 & -72.039 & 140.452 & -172.582 & 0.890 \\
\hline & -0.823 & 0.114 & 0.3 & 0.003 & 0.002 & & 0.062 & 0.084 & 0.075 & 1.252 & 1.628 & 2.363 & 0.005 \\
\hline \multirow[t]{2}{*}{ NGC 7099} & 325.092 & 8.458 & -185.19 & -0.698 & -7.302 & 0.007 & -2.989 & 2.644 & -6.164 & 0.453 & -86.896 & 108.702 & 0.424 \\
\hline & -23.18 & 0.09 & 0.17 & 0.050 & 0.050 & & 0.058 & 0.03 & 0.07 & 1.327 & 3.396 & 0.789 & 0.011 \\
\hline
\end{tabular}

TABLE II. This table represents the fundamental parameters of five GC samples.

\begin{tabular}{ccccc}
\hline \hline Cluster & Age(Gyr) & {$[\mathrm{Fe} / \mathrm{H}]$} & $(\mathrm{m}-\mathrm{M})$ & $E(B-V)$ \\
\hline Pal 1 & 8.0 & -0.65 & 15.25 & 0.50 \\
Pal 2 & 11.9 & $-1 . .42$ & 17.10 & 1.24 \\
NGC 1851 & 11.5 & -1.18 & 15.50 & 0.02 \\
NGC 7089 & 12.0 & -1.65 & 15.35 & 0.05 \\
NGC 7099 & 13.0 & -2.19 & 14.8 & 0.03 \\
\hline \hline
\end{tabular}

cee with nwalkers of 50 and nsteps of 2000 to estimate the dispersion profile of proper motion and calculated these parameters by using the emcee package [11].

\section{SUMMARY}

We have derived the mean proper motions and orbital parameters as well as the energy of five different clusters based on the compilation of different literature and Gaia EDR3. Our finding of proper motion is in good agreement with [1] but other parameters have been adopted from various literature [10] [5] [12]. In addition, we used a two-dimensional kernel density function for three compact clusters to find the peak of pixels for positions. The orbits were integrated for 5 Gyr backward using gala considering potential of MWPotential2014 which we can see in Fig. 3. Our conclusions are summarised as follows

- We estimated the median value of right ascension (RA), declination (DE), proper motion in right ascension (pmRA), and proper motion in declination (pmDE).

- We integrated the orbits of these clusters $5 \mathrm{Gyr}$ backward. Among five clusters, two clusters have high eccentricity, i.e. $e>0.7$. In general, the eccentricity greater than 0.7 belongs to the Gaia Enceladus [13]. We have also found that three clusters, NGC 7089, NGC 7099, and NGC 1851, exhibit retrograde motion $(L z>0)$ while two clusters, Pal 1 and Pal 2, exhibit prograde motion $(L z<0)$. This is convenient for the right-handed coordinate system. But our calculated apocentric and pericentric distances are largely differ from other literature. We have also studied the dispersion profile of proper motion with distance (arcmin) from the center of the clusters. We have found that the estimated values of eccentricity and other parameters have slightly differed while taking two different potential McMillan17 [14] and MWPotential2014 bovy2015galpy.

- We have found that the NGC 7099 is the oldest globular cluster age of $\sim 13 \mathrm{Gyr}$ with metallicity $[\mathrm{Fe} / \mathrm{H}]=2.19$, whereas the youngest globular cluster we find in our sample is Pal 1 with an age of $\sim 8$ Gyr, which is metal-rich. We examined the proper motion and dynamical properties of five globular clusters, NGC 7089, Pal2, NGC 7099, Pal1 and NGC 1851, with 1311, 45, 1640, 41, 1230 samples, respectively.

\section{ACKNOWLEDGMENTS}

This work has made use of data from the European Space Agency (ESA) mission Gaia (http://www.cosmos.eas.int/ gaia), processed by the Gaia Data Processing and Analysis Consortium (DPAC, http://ww.cosmos.eas. init/web/gaia /dpac/consortium) (Gaia Collaboration et al. 2016, 2018b). Funding for the DPAC has been provided by national institutions, in particular the institutions participating in the Gaia Multilateral Agreement. RKP acknowledges Tri-Chandra Multiple Campus, and Amrit Campus, Tribhuvan University, Nepal, for providing the research ground.

Software :matplotlib [15], numpy [12], scipy [16], jupyter [17], Astropy ( [18]; [19]), emcee [11], gala [20], sklearn [21]. 


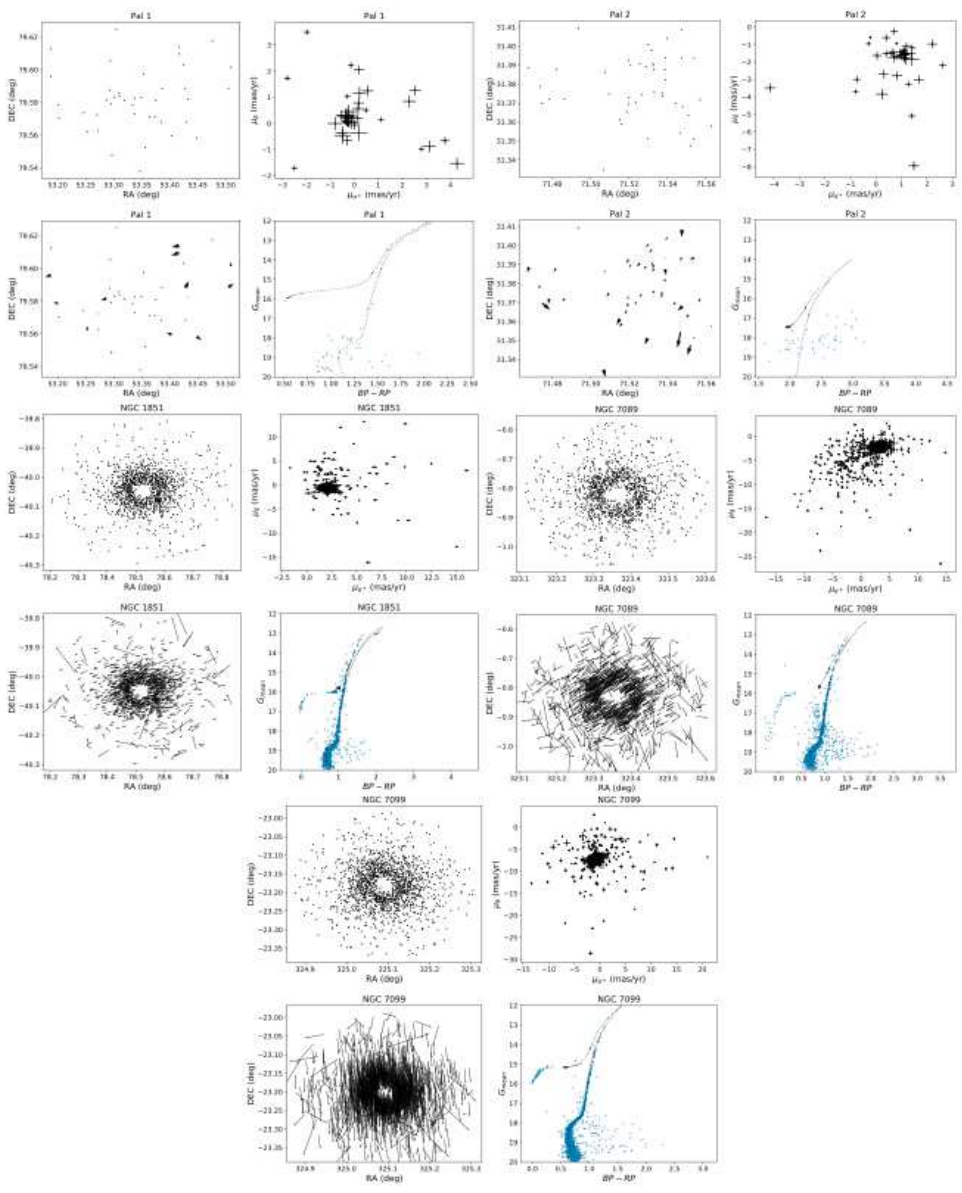

FIGURE 1. Overview of five cluster samples in position, proper motion, and color-magnitude diagram (CMD). The first top two panels represent various plots between RA and DEC, proper motions with error and distribution of the sample in RA and DEC with vector direction of proper motion, and isochrone fitting for two different clusters, Pal 1 and Pal2. Similarly, in the middle two planes, there are various plots for NGC 1851 and NGC 7089. The bottom two panels are present for NGC 7099. Here we have used PARSEC isochrone [22].
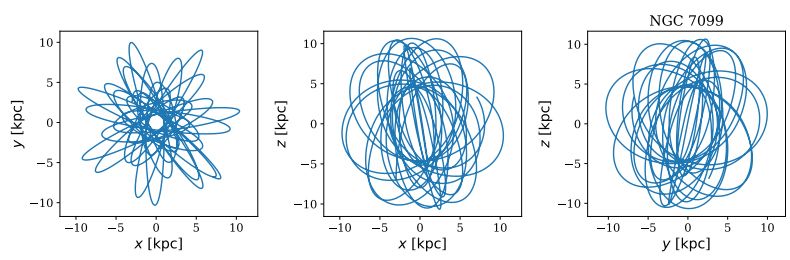

FIGURE 2. The sample orbits of NGC 7099 in our sample is presented. These integrated orbits are 5 Gyr year backward, assuming MWPotental14 potential [9]; [20]. We have taken solar motions with local circular velocity as a default of modified version-4 Astropy, 12.9, 245.6, $7.78 \mathrm{kms}^{-1}[8]$ and Galactocentric distance of $8.122 \mathrm{kpc}$ from the Sun.

\section{DATA AVAILABILITY}

This data used for this study is published by Gaia EDR3 archive which is found to be publicly available at https: https://gea.esac.esa.int/archive/. Some code to reproduce the analysis can be excessed via https://github.com/Galactic Dynamics-Oxford/GaiaTools

\section{EDITOR'S NOTE}

This manuscript was submitted to the Association of Nepali Physicists in America (ANPA) Conference 2021 for publication in the special issue of Journal of Nepal Physical Society. 

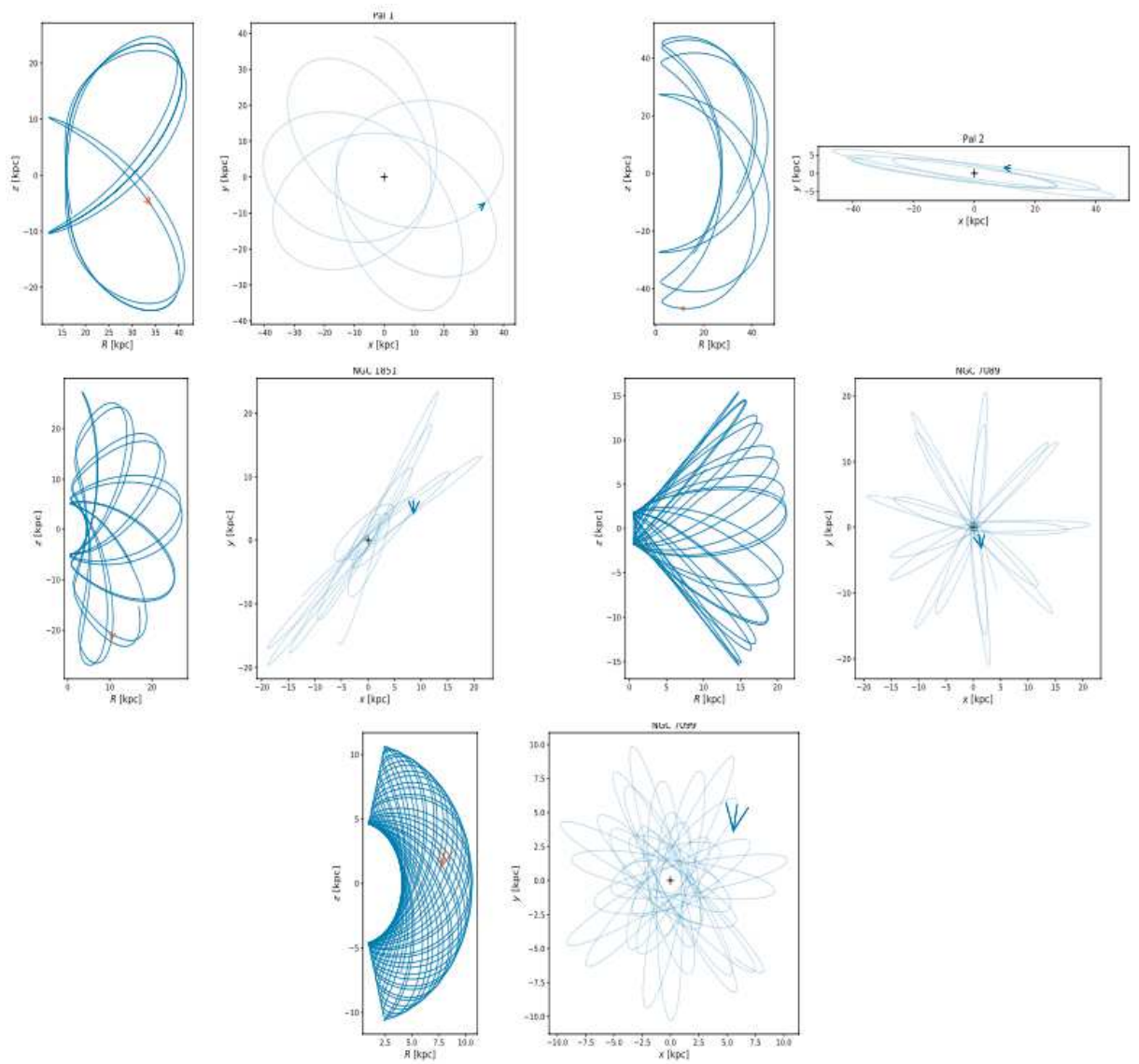

FIGURE 3. The orbit integration of all five Globular clusters. For the orbit integration, we have used gala gala with considering MWPotential2014 for 5 Gyr backyards. The grey plus symbol in the plots between $\mathrm{x}$ vs represents the Galactic center, and the arrowhead represents the direction of motion of the sample point on the orbit.

\section{REFERENCES}

1. E. Vasiliev and H. Baumgardt, Mon. Not. R. Astron. Soc. (2021).

2. H. Baumgardt and E. Vasiliev, Mon. Not. R. Astron. Soc. (2021).

3. A. G. Brown, A. Vallenari, T. Prusti, J. De Bruijne, C. Babusiaux, M. Biermann, O. Creevey, D. Evans, L. Eyer, A. Hutton, et al., Astron. Astrophys. 649, A1 (2021).

4. A. Brown et al., Astron. Astrophys. 616, A10 (2018).

5. H. Baumgardt, M. Hilker, A. Sollima, and A. Bellini, Mon. Not. . R. Astron. Soc. 482, 5138-5155 (2019).

6. E. Vitral, Mon. Not. R. Astrono. Soc. 504, 1355-1369 (2021).

7. L. Lindegren, S. Klioner, J. Hernández, A. Bombrun, M. RamosLerate, H. Steidelmüller, U. Bastian, M. Biermann, A. de Torres, E. Gerlach, et al., Astron. Astrophys. 649, A2 (2021).

8. R. Schönrich, J. Binney, and W. Dehnen, Mon. Not. . R. Astron. Soc. 403, 1829-1833 (2010).

9. J. Bovy, Astrophys. J. Supp. Ser. 216, 29 (2015).

10. W. E. Harris, Astron. J. 112, 1487 (1996).

11. D. Foreman-Mackey, D. W. Hogg, D. Lang, and J. Goodman, PASP 125, 306-312 (2013)

12. C. R. Harris, K. J. Millman, and S. J. van der Walt et al., Nature 585, 357-362 (2020)

13. R. P. Naidu, C. Conroy, A. Bonaca, B. D. Johnson, Y.-S. Ting, N. Caldwell, D. Zaritsky, and P. A. Cargile, Astrophys. J. 901, $48(2020)$
14. P. J. McMillan, Mon. Not. R. Astrono. Soc. , stw2759 (2016).

15. J. D. Hunter, Comput Sci Eng 9, 90-95 (2007).

16. P. Virtanen, R. Gommers, T. E. e. a. Oliphant, and SciPy 1.0 Contributors, Nat. Methods 17, 261-272 (2020)

17. T. Kluyver, B. Ragan-Kelley, and F. P. et al., in Positioning and Power in Academic Publishing: Players, Agents and Agendas, edited by F. Loizides and B. Schmidt (IOS Press, 2016) pp. 87 90.

18. Astropy Collaboration, T. P. Robitaille, and E. J. e. a. Tollerud, "Astropy: A community Python package for astronomy," 558, A33 (2013) 1307.6212 [astro-ph.IM]

19. Astropy Collaboration, A. M. Price-Whelan, B. M. Sipőcz, H. M. e. a. Günther, and Astropy Contributors, "," 156, 123 (2018) arXiv:1801.02634 [astro-ph.IM]

20. A. M. Price-Whelan, "Gala: A python package for galactic dynamics," J. Op. S. Soft.e 2 (2017), 10.21105/joss.00388

21. L. Buitinck, G. Louppe, and M. B. et al., in ECML PKDD Workshop: Languages for Data Mining and Machine Learning (2013) pp. $108-122$.

22. A. Bressan, P. Marigo, L. Girardi, B. Salasnich, C. Dal Cero, S. Rubele, and A. Nanni, Mon. Not. R. Astron. Soc. 427, 127-145 (2012). 